\title{
A CONTRACTION THEOREM IN MENGER SPACE
}

\author{
B. D. PANT AND SUNNY CHAUHAN
}

\begin{abstract}
The main purpose of this paper is to prove a common fixed point theorem satisfying a new contraction type condition without appeal to continuity in Menger space.
\end{abstract}

\section{Introduction}

The concept of probabilistic metric space was first introduced and studied by Menger [4], which is a generalization of the metric space and also the study of this space was expanded rapidly with the pioneering works of Schweizer and Sklar $[8,9]$. It is also of fundamental importance in probabilistic functional analysis, nonlinear analysis and applications [1].

In 1972, V.M. Sehgal and A.T. Bharucha-Reid [10] initiated the study of contraction mappings on probabilistic metric spaces. Several interesting and elegant results have been obtained by various authors in this direction. In 1986, Jungck [2] introduced the notion of compatible mappings in metric spaces. Mishra [5] extended the notion of compatibility to probabilistic metric spaces. And this condition has further been weakened by introducing the notion of weakly compatible mappings by Jungck and Rhoades [3]. The concept of weakly compatible mappings is most general as each pair of compatible mappings is weakly compatible but the reverse is not true. Recently, Singh and Jain [12] established a common fixed point theorem in Menger space using the concept of weak compatibility and compatibility of pair of self maps. For further information regarding this paper, we refer to $[6,7,11]$.

The purpose of this paper is to prove a common fixed point theorem for four weakly compatible mappings satisfying a new contraction type condition without appeal to continuity in Menger space.

Before start, we give some preliminaries.

Corresponding author: Sunny Chauhan.

Received January 20, 2010, revised April 26, 2010.

2000 Mathematics Subject Classification. Primary: 54H25, Secondary: 47H10.

Key words and phrases. Triangle function (t-norm), Menger space, Common fixed point, Compatible maps, Weakly compatible maps. 


\section{Preliminaries}

Definition 2.1 .([9]) A triangular norm $\mathrm{T}$ (shortly t-norm) is a binary operation on the unit interval $[0,1]$ such that for all $a, b, c, d \in[0,1]$ and the following conditions are satisfied:

(i) $\mathrm{T}(a, 1)=a$;

(ii) $\mathrm{T}(a, b)=\mathrm{T}(b, a)$;

(iii) $\mathrm{T}(a, b) \leq \mathrm{T}(c, d)$ for $a \leq c, b \leq d$;

(iv) $\mathrm{T}(\mathrm{T}(a, b), c)=\mathrm{T}(a, \mathrm{~T}(b, c))$;

Examples of t-norms are $\mathrm{T}(a, b)=\min \{a, b\}$ and $\mathrm{T}(a, b)=\max \{a+b-1,0\}$.

Definition 2.2.([9]) A mapping $F: \mathbb{R} \rightarrow \mathbb{R}^{+}$is called a distribution function if it is non-decreasing and left continuous with $\inf \{F(t): t \in \mathbb{R}\}=0$ and $\sup \{F(t): t \in \mathbb{R}\}=1$.

We shall denote by $\Delta_{+}$the set of all distribution functions defined on $[-\infty, \infty]$ while $\mathrm{H}$ will always denote the specific distribution function defined by

$$
H(t)=\left\{\begin{array}{lll}
0, & \text { if } & t \leq 0 \\
1, & \text { if } & t>0
\end{array}\right.
$$

If $X$ is a non-empty set, $F: X \times X \rightarrow \triangle_{+}$is called a probabilistic distance on $X$ and the value of $F$ at $(x, y) \in X \times X$ is represented by $F_{x, y}$.

Definition 2.3.([9]) The ordered pair $(X, F)$ is called a probabilistic metric space (shortly PMspace) if $X$ is a nonempty set and $F$ is a probabilistic distance satisfying the following conditions: for all $x, y, z \in X$ and $t, s>0$,

(i) $F_{x, y}(t)=1 \Leftrightarrow x=y$;

(ii) $F_{x, y}(0)=0$;

(iii) $F_{x, y}(t)=F_{y, x}(t)$;

(iv) if $F_{x, y}(t)=1$ and $F_{y, z}(s)=1$ then $F_{x, z}(t+s)=1$.

The ordered triple $(X, F, \mathrm{~T})$ is called a Menger space if $(X, F)$ is a PM-space, $\mathrm{T}$ is a t-norm and the following inequality holds:

(v) $F_{x, y}(t+s) \geq \mathrm{T}\left(F_{x, z}(t), F_{z, y}(s)\right)$, for all $x, y, z \in X$ and $t, s>0$.

Every metric space $(X, d)$ can always be realized as a PM space by considering $F: X \times X \rightarrow$ $\triangle_{+}$defined by $F_{x, y}(t)=H(t-d(x, y))$ for all $x, y \in X$. 
Definition 2.4. ([9]) Let $(X, F, \mathrm{~T})$ be a Menger space with continuous t-norm.

(i) A sequence $\left\{x_{n}\right\}$ in $X$ is said to be converge to a point $x$ in $X$ if and only if for every $\epsilon>0$ and $\lambda \in(0,1)$, there exists an integer $N$ such that $F_{x_{n}, x}(\epsilon)>1-\lambda$ for all $n \geq N$.

(ii) A sequence $\left\{x_{n}\right\}$ in $X$ is said to be Cauchy if for every $\epsilon>0$ and $\lambda \in(0,1)$, there exists an integer $N$ such that $F_{x_{n}, x_{m}}(\epsilon)>1-\lambda$ for all $n, m \geq N$.

(iii) A Menger space in which every Cauchy sequence is convergent is said to be complete.

Definition 2.5.([5]) Self maps $A$ and $B$ of a Menger space $(X, F, T)$ are said to be compatible if and only if $F_{A B x_{n}, B A x_{n}}(t) \rightarrow 1$ for all $t>0$, whenever $\left\{x_{n}\right\}$ is a sequence in $X$ such that $A x_{n}, B x_{n} \rightarrow x$ for some $x$ in $X$.

Definition 2.6.([12]) Self maps $A$ and $B$ of a Menger space $(X, F, T)$ are said to be weakly compatible (or coincidentally commuting) if they commute at their coincidence points, that is, if $A x=B x$ for some $x \in X$, then $A B x=B A x$.

Remark 2.1 .([12]) If self maps $A$ and $B$ of a Menger space $(X, F, \mathrm{~T})$ are compatible then they are weakly compatible but the converse is not true. Therefore the concept of weak compatibility is more general than that of compatibility.

The following is an example of pair of self maps in a Menger space which are weakly compatible but not compatible.

Example 2.1. Let $(X, d)$ be a metric space defined by $d(x, y)=|x-y|$, where $X=[0,6]$ and $(X, F, \mathrm{~T})$ be the induced Menger space with $F_{x, y}(t)=\frac{t}{t+d(x, y)}$, for all $t>0$. We define self maps $A$ and $B$ as follows:

$$
A(x)=\left\{\begin{array}{ll}
6-x, & \text { if } \quad 0 \leq x<3 ; \\
6, & \text { if } 3 \leq x \leq 6 .
\end{array} \quad B(x)=\left\{\begin{array}{lll}
x, & \text { if } & 0 \leq x<3 \\
6, & \text { if } & 3 \leq x \leq 6
\end{array}\right.\right.
$$

Taking $x_{n}=3-\frac{1}{n}$. We get $A x_{n}=3+\frac{1}{n}, B x_{n}=3-\frac{1}{n}$. Thus, $A x_{n} \rightarrow 3, B x_{n} \rightarrow 3$. Hence $x=3$. Further $A B x_{n}=3+\frac{1}{n}, B A x_{n}=6$. Now; $\lim _{n \rightarrow \infty} F_{A B x_{n}, B A x_{n}}(t)=\lim _{n \rightarrow \infty} F_{3+\frac{1}{n}, 6}(t)=\frac{t}{t+3}<1$, for all $t>0$. Hence $(A, B)$ is not compatible.

Coincidence points of $A$ and $B$ are in [3,6]. Now for any $x \in[3,6] . A x=B x=6$ and $A B(x)=A(6)=6=B(6)=B A(x)$. Thus $(A, B)$ is weakly compatible.

Lemma 2.1 ( $(6,11])$ Let $(X, F, T)$ be a Menger probabilistic metric space and define $E_{\lambda, F}: X^{2} \rightarrow$ $\mathbb{R}^{+} \cup\{0\}$ by 


$$
E_{\lambda, F}(x, y)=\inf \left\{t>0: F_{x, y}(t)>1-\lambda\right\}
$$

for each $\lambda \in(0,1)$ and $x, y \in X$. Then we have

(i) For any $\mu \in(0,1)$ there exists $\lambda \in(0,1)$ such that

$$
E_{\mu, F}\left(x_{1}, x_{n}\right) \leq E_{\lambda, F}\left(x_{1}, x_{2}\right)+\ldots+E_{\lambda, F}\left(x_{n-1}, x_{n}\right),
$$

for any $x_{1}, \ldots, x_{n} \in X$.

(ii) The sequence $\left\{x_{n}\right\}_{n \in N}$ is convergent with respect to Menger probabilistic metric $F$ if and only if $E_{\lambda, F}\left(x_{n}, x\right) \rightarrow 0$. Also the sequence $\left\{x_{n}\right\}$ is a Cauchy sequence with respect to Menger probabilistic metric $F$ if and only if it is a Cauchy sequence with $E_{\lambda, F}$.

\section{Proof.}

(i) For every $\mu \in(0,1)$, we can find a $\lambda \in(0,1)$ such that

$$
\mathrm{T}^{n-1}(1-\lambda, \ldots, 1-\lambda)>1-\mu .
$$

By the triangular inequality, we have

$$
\begin{aligned}
& F_{x_{1}, x_{n}}\left(E_{\lambda, F}\left(x_{1}, x_{2}\right)+\ldots+E_{\lambda, F}\left(x_{n-1}, x_{n}\right)+n \delta\right) \\
& \quad \geq \mathrm{T}^{n-1}\left(F_{x_{1}, x_{2}}\left(E_{\lambda, F}\left(x_{1}, x_{2}\right)+\delta\right), \ldots, F_{x_{n-1}, x_{n}}\left(E_{\lambda, F}\left(x_{n-1}, x_{n}\right)+\delta\right)\right) \\
& \quad \geq \mathrm{T}^{n-1}(1-\lambda, \ldots, 1-\lambda)>1-\mu,
\end{aligned}
$$

for every $\delta>0$, which implies that

$$
E_{\mu, F}\left(x_{1}, x_{n}\right) \leq E_{\lambda, F}\left(x_{1}, x_{2}\right)+\ldots+E_{\lambda, F}\left(x_{n-1}, x_{n}\right)+n \delta
$$

Since $\delta>0$ is arbitrary, we have

$$
E_{\mu, F}\left(x_{1}, x_{n}\right) \leq E_{\lambda, F}\left(x_{1}, x_{2}\right)+\ldots+E_{\lambda, F}\left(x_{n-1}, x_{n}\right) .
$$

(i) (ii)] We have $F_{x_{n}, x}(\eta)>1-\lambda \Leftrightarrow E_{\lambda, F}\left(x_{n}, x\right)<\eta$, for every $\eta>0$.

\section{Result}

Theorem 3.1. Let $A, L, M$ and $S$ be self maps on a complete Menger space $(X, F, T)$ and satisfy the following conditions:

(a) $L(X) \subseteq S(X), M(X) \subseteq A(X)$;

(b) $S(X)$ and $A(X)$ are complete subspaces of $X$; 
(c) There exists a constant $k \in(0,1)$ such that

$$
\min \left\{F_{L x, M y}(k t), F_{S y, L x}(k t)\right\}+\gamma F_{S y, M y}(k t) \geq\left[\alpha F_{A x, L x}(t)+\beta F_{A x, S y}(t)\right],
$$

for all $x, y \in X$ and $t>0$ where $0<\alpha, \beta<1$ and $0 \leq \gamma<1$ such that $\alpha+\beta-\gamma=1$;

(d) The pairs $\{L, A\}$ and $\{M, S\}$ are weakly compatible;

In addition assume that

$$
E_{\lambda, F}(x, y)=\inf \left\{t>0: F_{x, y}(t)>1-\lambda\right\}
$$

for each $\lambda \in(0,1)$ and $x, y \in X$.

Then $A, L, M$ and $S$ have a unique common fixed point in $X$.

Proof. Let $x_{0}$ be an arbitrary element in $X$. By (a), there exist $x_{1}, x_{2} \in X$ such that $L x_{0}=S x_{1}$ and $M x_{1}=A x_{2}$. Inductively, we construct sequences $\left\{x_{n}\right\}$ and $\left\{y_{n}\right\}$ in $X$ such that $L x_{2 n}=$ $S x_{2 n+1}=y_{2 n}$ and $M x_{2 n+1}=A x_{2 n+2}=y_{2 n+1}$ for $n=0,1,2, \ldots$.

Putting $x=x_{2 n}$ and $y=x_{2 n+1}$ in (c), then we get

$$
\begin{aligned}
& \min \left\{F_{L x_{2 n}, M x_{2 n+1}}(k t), F_{S x_{2 n+1}, L x_{2 n}}(k t)\right\}+\gamma F_{S x_{2 n+1}, M x_{2 n+1}}(k t) \\
& \quad \geq\left[\alpha F_{A x_{2 n}, L x_{2 n}}(t)+\beta F_{A x_{2 n}, S x_{2 n+1}}(t)\right] \\
& \min \left\{F_{y_{2 n}, y_{2 n+1}}(k t), F_{y_{2 n}, y_{2 n}}(k t)\right\}+\gamma F_{y_{2 n}, y_{2 n+1}}(k t) \geq\left[\alpha F_{y_{2 n-1}, y_{2 n}}(t)+\beta F_{y_{2 n-1}, y_{2 n}}(t)\right], \\
& \min \left\{F_{y_{2 n}, y_{2 n+1}}(k t), 1\right\}+\gamma F_{y_{2 n}, y_{2 n+1}}(k t) \geq(\alpha+\beta) F_{y_{2 n-1}, y_{2 n}}(t), \\
& F_{y_{2 n}, y_{2 n+1}}(k t)+\gamma F_{y_{2 n}, y_{2 n+1}}(k t) \geq(\alpha+\beta) F_{y_{2 n-1}, y_{2 n}}(t) \\
& (1+\gamma) F_{y_{2 n}, y_{2 n+1}}(k t) \geq(1+\gamma) F_{y_{2 n-1}, y_{2 n}}(t) \\
& F_{y_{2 n}, y_{2 n+1}}(k t) \geq F_{y_{2 n-1}, y_{2 n}}(t)
\end{aligned}
$$

Similarly,

$$
F_{y_{2 n+1}, y_{2 n+2}}(k t) \geq F_{y_{2 n}, y_{2 n+1}}(t) .
$$

Therefore, for all $n$, we have,

$$
F_{y_{n}, y_{n+1}}(k t) \geq F_{y_{n-1}, y_{n}}(t) .
$$

Consequently,

$$
F_{y_{n}, y_{n+1}}(t) \geq F_{y_{n-1}, y_{n}}\left(\frac{t}{k}\right) .
$$

By repeated application of above inequality, we get

$$
F_{y_{n}, y_{n+1}}(t) \geq F_{y_{n-1}, y_{n}}\left(\frac{t}{k}\right) \geq \cdots \geq F_{y_{0}, y_{1}}\left(\frac{t}{k^{n}}\right),
$$


for $n=1,2,3, \ldots$ which implies that

$$
\begin{aligned}
E_{\lambda, F}\left(y_{n}, y_{n+1}\right) & =\inf \left\{t>0: F_{y_{n}, y_{n+1}}(t)>1-\lambda\right\} \\
& \leq \inf \left\{t>0: F_{y_{0}, y_{1}}\left(\frac{t}{k^{n}}\right)>1-\lambda\right\} \\
& =k^{n} \inf \left\{t>0: F_{y_{0}, y_{1}}(t)>1-\lambda\right\} \\
& =k^{n} E_{\lambda, F}\left(y_{0}, y_{1}\right),
\end{aligned}
$$

for every $\lambda \in(0,1)$.

Now, we show that $\left\{y_{n}\right\}$ is a Cauchy sequence. For every $\mu \in(0,1)$, there exists $\psi \in(0,1)$ such that, for $m \geq n$,

$$
\begin{aligned}
E_{\mu, F}\left(y_{n}, y_{m}\right) & =E_{\psi, F}\left(y_{m-1}, y_{m}\right)+E_{\psi, F}\left(y_{m-2}, y_{m-1}\right)+\ldots+E_{\psi, F}\left(y_{n}, y_{n+1}\right) \\
& =E_{\psi, F}\left(y_{0}, y_{1}\right) \sum_{i=n}^{m-1} k^{i} \rightarrow 0
\end{aligned}
$$

as $m, n \rightarrow \infty$. Thus by Lemma $2.1,\left\{y_{n}\right\}$ is a Cauchy sequence in $X$. Since $X$ is complete. Therefore $\left\{y_{n}\right\}$ converges to $z \in X$. That is

$$
\lim _{n \rightarrow \infty} y_{n}=\lim _{n \rightarrow \infty} L x_{2 n}=\lim _{n \rightarrow \infty} S x_{2 n+1}=\lim _{n \rightarrow \infty} M x_{2 n+1}=\lim _{n \rightarrow \infty} A x_{2 n}=z .
$$

Since $S(X)$ and $A(X)$ are complete subspaces of $X$ then there exist $v, w \in X$ such that $S(v)=z$ and $A(w)=z$. Now we prove that $S(v)=M(v)=z$ and $A(w)=L(w)=z$.

Put $x=x_{2 n}$ and $y=v$ in (c), then we have

$$
\min \left\{F_{L x_{2 n}, M v}(k t), F_{S v, L x_{2 n}}(k t)\right\}+\gamma F_{S \nu, M v}(k t) \geq\left[\alpha F_{A x_{2 n}, L x_{2 n}}(t)+\beta F_{A x_{2 n}, S v}(t)\right],
$$

as $n \rightarrow \infty$, we have

$$
\begin{aligned}
& \min \left\{F_{z, M v}(k t), F_{z, z}(k t)\right\}+\gamma F_{z, M v}(k t) \geq\left[\alpha F_{z, z}(t)+\beta F_{z, z}(t)\right], \\
& \min \left\{F_{z, M v}(k t), 1\right\}+\gamma F_{z, M v}(k t) \geq(\alpha+\beta), \\
& F_{z, M v}(k t)+\gamma F_{z, M v}(k t) \geq(\alpha+\beta), \\
& (1+\gamma) F_{z, M v}(k t) \geq(\alpha+\beta), \\
& F_{z, M v}(k t) \geq\left(\frac{\alpha+\beta}{1+\gamma}\right)=1,
\end{aligned}
$$

for $k \in(0,1)$ and $t>0$. Thus, we have $z=M v$. Therefore, $z=M v=S v$.

Now, we put $x=w$ and $y=x_{2 n+1}$ in (c), then we have $\min \left\{F_{L w, M x_{2 n+1}}(k t), F_{S x_{2 n+1}, L w}(k t)\right\}+\gamma F_{S x_{2 n+1}, M x_{2 n+1}}(k t) \geq\left[\alpha F_{A w, L w}(t)+\beta F_{A w, S x_{2 n+1}}(t)\right]$, 
as $n \rightarrow \infty$, we have

$$
\begin{aligned}
& \min \left\{F_{L w, z}(k t), F_{z, L w}(k t)\right\}+\gamma F_{z, z}(k t) \geq\left[\alpha F_{z, L w}(t)+\beta F_{z, z}(t)\right], \\
& F_{L w, z}(k t)+\gamma \geq\left[\alpha F_{z, L w}(t)+\beta\right], \\
& F_{L w, z}(k t) \geq \alpha F_{z, L w}(t)+(\beta-\gamma) \geq \alpha F_{z, L w}(k t)+(\beta-\gamma), \\
& F_{L w, z}(k t) \geq\left(\frac{\beta-\gamma}{1-\alpha}\right)=1,
\end{aligned}
$$

for $k \in(0,1)$ and $t>0$. Thus, we have $z=L w$. Therefore, $z=L w=A w$.

It is given that the pairs $\{L, A\}$ and $\{M, S\}$ are weakly compatible, then $L(A(w))=A(L(w))$, that is $L z=A z$ and $M(S(\nu))=S(M(v))$, that is $M z=S z$. Now we prove that $L z=A z=z$ and $M z=S z=z$.

Put $x=z$ and $y=x_{2 n+1}$ in (c), then we have

$$
\min \left\{F_{L z, M x_{2 n+1}}(k t), F_{S x_{2 n+1}, L z}(k t)\right\}+\gamma F_{S x_{2 n+1}, M x_{2 n+1}}(k t) \geq\left[\alpha F_{A z, L z}(t)+\beta F_{A z, S x_{2 n+1}}(t)\right],
$$

as $n \rightarrow \infty$, we get

$$
\begin{aligned}
& \min \left\{F_{A z, z}(k t), F_{z, A z}(k t)\right\}+\gamma F_{z, z}(k t) \geq\left[\alpha F_{A z, A z}(t)+\beta F_{A z, z}(t)\right], \\
& F_{A z, z}(k t)+\gamma \geq\left[\alpha+\beta F_{A z, z}(t)\right], \\
& F_{A z, z}(k t) \geq \beta F_{A z, z}(t)+(\alpha-\gamma) \geq \beta F_{A z, z}(k t)+(\alpha-\gamma), \\
& F_{A z, z}(k t) \geq\left(\frac{\alpha-\gamma}{1-\beta}\right)=1,
\end{aligned}
$$

for $k \in(0,1)$ and $t>0$. Thus, we have $A z=z$. Therefore, $z=L z=A z$.

Now, we put $x=x_{2 n}$ and $y=z$ in (c), then we have

$$
\begin{aligned}
& \min \left\{F_{L x_{2 n}, M z}(k t), F_{S z, L x_{2 n}}(k t)\right\}+\gamma F_{S z, M z}(k t) \geq\left[\alpha F_{A x_{2 n}, L x_{2 n}}(t)+\beta F_{A x_{2 n}, S z}(t)\right], \\
& \min \left\{F_{z, S z}(k t), F_{S z, z}(k t)\right\}+\gamma F_{S z, S z}(k t) \geq\left[\alpha F_{z, z}(t)+\beta F_{z, S z}(t)\right], \\
& F_{z, S z}(k t)+\gamma \geq\left[\alpha+\beta F_{z, S z}(t)\right], \\
& F_{z, S z}(k t) \geq \beta F_{z, S z}(t)+(\alpha-\gamma) \geq \beta F_{z, S z}(k t)+(\alpha-\gamma), \\
& F_{z, S z}(k t) \geq\left(\frac{\alpha-\gamma}{1-\beta}\right)=1,
\end{aligned}
$$

for $k \in(0,1)$ and $t>0$. Thus, we have $z=S z$. Therefore, $z=M z=S z$.

Now combine all the results then we get $z=A z=L z=M z=S z$. That is $z$ is the common fixed point of $A, L, M$ and $S$.

Uniqueness: Let $u(u \neq z)$ be another common fixed point of $A, L, M$ and $S$. Put $x=z$ and $y=u$ in (c), then we get

$$
\min \left\{F_{L z, M u}(k t), F_{S u, L z}(k t)\right\}+\gamma F_{S u, M u}(k t) \geq\left[\alpha F_{A z, L z}(t)+\beta F_{A z, S u}(t)\right],
$$


which implies that,

$$
\begin{aligned}
& \min \left\{F_{z, u}(k t), F_{u, z}(k t)\right\}+\gamma F_{u, u}(k t) \geq\left[\alpha F_{z, z}(t)+\beta F_{z, u}(t)\right], \\
& F_{z, u}(k t)+\gamma \geq\left[\alpha+\beta F_{z, u}(t)\right], \\
& F_{z, u}(k t) \geq \beta F_{z, u}(t)+(\alpha-\gamma) \geq \beta F_{z, u}(k t)+(\alpha-\gamma), \\
& F_{z, u}(k t) \geq\left(\frac{\alpha-\gamma}{1-\beta}\right)=1,
\end{aligned}
$$

for $k \in(0,1)$ and $t>0$. Thus, we have $z=u$. Therefore, $z$ is the unique common fixed point of the self maps $A, L, M$ and $S$.

The following example illustrates Theorem 3.1.

Example 3.1. Let $X=[0,30]$ with the metric d defined by $d(x, y)=|x-y|$ and for each $t \in[0,1]$ define

$$
F_{x, y}(t)=\left\{\begin{array}{lll}
\frac{t}{t+|x-y|}, & \text { if } & t>0 \\
0, & \text { if } & t=0 .
\end{array}\right.
$$

for all $x, y \in X$. Clearly $(X, F, \mathrm{~T})$ is a complete Menger space, where $\mathrm{T}$ is a continuous t-norm. Define $A, L, M$ and $S: X \rightarrow X$ by

$$
\begin{array}{ll}
A(x)=\left\{\begin{array}{ll}
0, & \text { if } \quad x=0 ; \\
12, & \text { if } \quad 0<x \leq 15 ; \\
x-9, & \text { if } 15<x \leq 30 .
\end{array} \quad S(x)=\left\{\begin{array}{lll}
0, & \text { if } \quad x=0 ; \\
6, & \text { if } \quad 0<x \leq 15 ; \\
x-6, & \text { if } \quad 15<x \leq 30 .
\end{array}\right.\right. \\
L(x)=\left\{\begin{array}{lll}
0, & \text { if } \quad x=0 ; \\
6, & \text { if } & 0<x \leq 30 .
\end{array}\right. & M(x)=\left\{\begin{array}{lll}
0, & \text { if } & x=0 ; \\
9, & \text { if } & 0<x \leq 30 .
\end{array}\right.
\end{array}
$$

Then $A, L, M$ and $S$ satisfy all the conditions of Theorem 3.1 with $k \in(0,1)$ and have a unique common fixed point $0 \in X$. It may be noted in this example that the mappings $L$ and A commute at coincidence point $0 \in X$. So $L$ and $A$ are weakly compatible maps. Similarly, $M$ and $S$ are weakly compatible maps. To see the pairs $\{L, A\}$ and $\{M, S\}$ are not compatible, let us consider a sequence $\left\{x_{n}\right\}$ defined as $x_{n}=15+\frac{1}{n}, n \geq 1$, then $x_{n} \rightarrow 15$ as $n \rightarrow \infty$. Then $\lim _{n \rightarrow \infty} L x_{n}=6, \lim _{n \rightarrow \infty} A x_{n}=6{\text { but } \lim _{n \rightarrow \infty} F_{L A x_{n}}, A L x_{n}}(t)=\frac{t}{t+|6-12|} \neq 1$. Thus the pair $\{L, A\}$ is not compatible. Also $\lim _{n \rightarrow \infty} M x_{n}=9, \lim _{n \rightarrow \infty} S x_{n}=9$ but $\lim _{n \rightarrow \infty} F_{M S x_{n}}, S M x_{n}(t)=$ $\frac{t}{t+|9-6|} \neq 1$. So the pair $\{M, S\}$ is not compatible. All the mappings involved in this example are discontinuous even at the common fixed point $x=0$.

On taking $A=S$ and $L=M$ in Theorem 3.1, then we get the following:

Corollary 3.1. Let $A$ and $L$ be self maps on a complete Menger space $(X, F, T)$ and satisfy the following conditions: 
(a) $L(X) \subseteq A(X)$;

(b) $A(X)$ is a complete subspace of $X$;

(c) There exists a constant $k \in(0,1)$ such that

$$
\min \left\{F_{L x, L y}(k t), F_{A y, L x}(k t)\right\}+\gamma F_{A y, L y}(k t) \geq\left[\alpha F_{A x, L x}(t)+\beta F_{A x, A y}(t)\right],
$$

for all $x, y \in X$ and $t>0$ where $0<\alpha, \beta<1$ and $0 \leq \gamma<1$ such that $\alpha+\beta-\gamma=1$;

(d) The pair $\{L, A\}$ is weakly compatible;

In addition assume that

$$
E_{\lambda, F}(x, y)=\inf \left\{t>0: F_{x, y}(t)>1-\lambda\right\}
$$

for each $\lambda \in(0,1)$ and $x, y \in X$.

Then $A$ and L have a unique common fixed point in X.

\section{Acknowledgements}

The authors are thankful to the Editorial Committee's member Prof. Shou-Jen Hu for his useful suggestions and the referee for his critical remarks to improve the manuscript.

\section{References}

[1] S. S. Chang, Y. J. Cho and S. M. Kang, Nonlinear Operator Theory in Probabilistic Metric Spaces, Nova Science Publishers, Huntington, USA, 2001.

[2] G. Jungck, Compatible mappings and common fixed pointsZ, Internat. J. Math. \& Math. Sci., 9 (1986), 771773.

[3] G. Jungck and B.E. Rhoades, Fixed points for set valued functions without continuity, Indian J. Pure Appl. Math., 29 (1998), 227-238.

[4] K. Menger, Statistical metric, Proc. Nat. Acad. (USA) 28 (1942), 535-537.

[5] S. N. Mishra, Common fixed points of compatible mappings in probabilistic metric spaces, Math. Japon., 36 (1991), 283-289.

[6] D. O'Regan, R. Saadati, Nonlinear contraction theorems in probabilistic spaces, Appl. Math. Comput., 195 (2008), 86-93.

[7] B. D. Pant and S. Chauhan, A contraction theorem in Menger space using weak compatibility, Int. J. Math. Sci. \& Engg. Appls., 4 (2010), 177-186.

[8] B. Schweizer and A. Sklar, Statistical metric spaces, Pacific J. Math., 10 (1960), 313-334.

[9] B. Schweizer and A. Sklar, Probabilistic Metric Spaces, Elsevier, North-Holland, New York, 1983.

[10] V. M. Sehgal and A. T. Bharucha-Reid, Fixed point of contraction mappings on probabilistic metric spaces, Math. System Theory, 6 (1972), 97-102.

[11] S. Shakeri, A contraction theorem in Menger probabilistic metric spaces, J. Nonlinear Sci. Appl., 1 (2008), 189193. 
[12] B. Singh and S. Jain, A fixed point theorem in Menger space through weak compatibility, J. Math. Anal. Appl., 301 (2005), 439-448.

Principal, Government Degree College, Champawat, (Uttarakhand), India.

E-mail: badridatt.pant@gmail.com

R. H. Government Postgraduate College, Kashipur, 244 713, U.S. Nagar, (Uttarakhand), India.

E-mail: sun.gkv@gmail.com 